Case Report

\title{
Left Hemineglect After Ischemic Stroke to the Left Brain Hemisphere: A Case Study
}

\author{
Malkhaz Makashvili ${ }^{1,2,}$, Salome Nikoleishvili ${ }^{2}$ \\ ${ }^{1}$ Faculty of Arts and Sciences, Ilia State University, Tbilisi, Georgia \\ ${ }^{2}$ Students' Psycho-Physiological Research Center, Ilia University, Tbilisi, Georgia
}

Email address:

malkhaz_makashvili@iliauni.edu.ge (M. Makashvili)

${ }^{*}$ Corresponding author

\section{To cite this article:}

Malkhaz Makashvili, Salome Nikoleishvili. Left Hemineglect After Ischemic Stroke to the Left Brain Hemisphere: A Case Study. American Journal of Applied Psychology. Vol. 6, No. 4, 2017, pp. 83-87. doi: 10.11648/j.ajap.20170604.16

Received: December 25, 2016; Accepted: January 5, 2017; Published: October 18, 2017

\begin{abstract}
Patient, male, 67, was diagnosed as having ischemic degenerative changes in the fronto-parietal cortical area of the left hemisphere due to the occlusion of the middle cerebral artery. Clinical picture was characterized by right-sided hemiparesis in the upper right shoulder and relative weakness in the right leg, as well as motor aphasia. Patient was tested on hemineglect (HN) after 2 years of the initial stroke. No signs of HN were revealed in line bisection and cancelation tasks, as well as in copying the clock and Rey-Osterrieth figure from a sample. Drawing clock and Rey-Osterrieth figure from memory was characterized by omission of details from the left side of figures, suggesting left $\mathrm{HN}$ at a representational level.
\end{abstract}

Keywords: Left Hemineglect, Left Hemisphere, Ischemic Stroke

\section{Introduction}

Hemineglect - HN (Unilateral neglect, Unilateral inattention,) is diagnosed when patient is unable to pay attention to either right or left half of the space, contralateral to the side of brain lesion. Thi s is lateralized disorder with a characteristic failure to explore or react to stimuli presented in contralesional space $[6,18,20]$.

It does not necessarily mean that all stimuli on the side contralateral to the brain lesion should be ignored. Rather stimuli on the extreme contralateral side have a higher probability of being ignored while stimuli closer to the body midline have a lower probability of being ignored [30].

$\mathrm{HN}$ is common in patients with the damage to the right brain hemisphere. HN sufferers do not eat from the contralesional left part of the dish, leave face unshaved on the left side. When walking in the hospital or at home, patient may bump into furniture, situated on her/his left, and may fail to respond to a left-sided bystander [2]. When tested for HN, patients omit elements to their left when copying simple objects like cube or flower, drawing a clock face, and cancelling targets among distracters. They also tend to error to the right when asked to bisect a horizontal line [28]. When required to find and cancel (mark with a pen) target items distributed on sheet of paper, HN patients fail to cancel items in the side, contralateral to brain lesion [15].

Right brain hemisphere is suggested dominant in directing attention towards the opposite left side of hemispace [17]. Left hemisphere controls the right hemispace and in cases of the left-hemispheric lesion patients display right-sided $\mathrm{HN}[3$, 25].

To explain the origin of $\mathrm{HN}$, hemispheric rivalry model holds that hemispheres compete with each other for directing attention toward contralateral space. Dominant right hemisphere inhibits the left one. After right hemispheric lesion, left hemisphere is released from inhibition, becomes hyperactive in directing attention to the right part of space and this results in left-sided neglect. In turn, right-sided neglect after left-hemispheric lesion results from the overactivation of the right hemisphere, released form the inhibitory influence of the left hemisphere [12, 23, 24]. Compensation hypothesis $[16,18]$ suggests, that right hemisphere directs attention to both, ipsilateral as well as contralateral stimuli, while the left hemisphere organizes attention primarily to the 
contralateral stimulus. Damage to the right hemisphere and consequent hypoactivity of the right half-brain results in left-sided neglect and ipsilesional attentional bias.

However, hemispheric rivalry model is based on inhibitory interhemispheric interaction, while more recent studies suggest hemispheres to exert both, inhibitory as well as excitatory influence on each other [8,9]. Moreover, both, hemispheric rivalry model, as well as compensation hypothesis are applicable only to cases of contralesional neglect and can not explain the incidence of ipsilesional neglect. In fact, right hemispheric lesion may cause ipsilesional $\mathrm{HN}[10,22,26]$ as well as damage to the left hemisphere may result in left-sided $\mathrm{HN}[11,21]$.

Evidently, existing theoretical explanations of $\mathrm{HN}$ are not sufficient for clear understanding of the origin of $\mathrm{HN}$. We report here on the left-sided hemineglect in case of the damage to the left hemisphere in the hope, that extension of the research in this direction may help in better understanding of the nature of $\mathrm{HN}$.

\section{Material and Methods}

Patient, male, 67, with higher university education, suffered from ischemic stroke in 2013. CT scan performed in the acute phase showed a left brain lesion in the territory of the middle cerebral artery involving parietal and frontal lobes of the left hemisphere. The clinical picture in the acute phase was characterized by right-sided hemiparesis in the upper right shoulder and relative weakness in the right leg, as well as complete motor aphasia. Sensory aphasia was expressed in inability to understand complexs instructions. At the same time, patient was able to correctly point to the written names of the household as well as to the written names of things of everyday use in response to the question like: which one is a name of your daughter? Which word is a "cup"? Neglect was not directly investigated in the acute phase. MRI scan after one month of initial stroke revealed atrophy of cortical tissue in the fronto-parietal area, moderate dilation of the left lateral ventricle and spared ganglia within the white matter of the brain hemispheres. We examined the patient after 2 years of the initial stroke. Clinical examination at that moment diagnosed right-sided hemiparesis in the upper shoulder, weakness in the right leg and motor aphasia. No signs of hemianopia were revealed. It was impossible to assess handedness of the patient because of hemiparesis, however household witnessed that patient was right-hander and no sign of left-handedness was registered in nearest ancestry. Consent for testing was obtained from patient and his family. Study was conducted on compliance with the latest version of the Declaration of Helsinki.

Examination was performed in 3 sessions, with intersession period of one month.

In every session patient was required to perform the pen and pencil tasks, as following: 1. Line bisection (LB), 2. Bell test (BT), 3. Copying the clock from a sample (CC) and drawing a clock by memory (CM), 4. Copying the Rey-Osterrieth figure (ROC), 5. Drawing Rey-Osterrieth figure by memory (ROM). LB, BT and CC performance was examined three times, with one month interval between sessions.

In the LB task the patient was asked to mark the center of lines. The series of 10 horizontal lines of different length were presented one by one. More than $6 \mathrm{~mm}$ displacement of the bisection mark from the center in at least 3 cases out of the 10 was considered an error, suggesting HN [27]. Each error scored 1, maximum scores 10 . In the BT the patient was asked to circle all 35 bells embedded within 280 distracters [figures of animals, house etc.] in a 15-85 inch page placed directly in front of him at the midline. The total number of circled targets was recorded. Omission of one element scored 1 , maximum score 35 . Diagnosis of $\mathrm{HN}$ is considered when the minimum number of omissions in a BT ranges from 13 to $15 \%$ of the targets to be cancelled [14]. In the CC the patient was required to make a copy from a penciled clock within 10 minutes. After task completion, in $\mathrm{CM}$, patient was required to reproduce a clock from memory. In both, the $\mathrm{CC}$ as well as in the $\mathrm{CM}$ each omitted element [12 digits and 2 arms, 14 in total] scored 1, maximum score 14. In ROC patient was asked to make a copy of the Rey-Osterrieth sample figure. The time limit was 30 minutes. After task completion, in ROM, patient was required to reproduce a figure from memory. The sample elements were numbered to evaluate possible omissions and displacement of elements in ROC and ROM. Assessment of task performance (number of elements omitted, displaced and distorted as well as 5 level assessment of drawing stile) was done according to widely accepted method, as described in [29]. To diagnose HN in CC, CM, ROC and ROM we followed the criterion reported in [19]. Catherine Bergego Scale (CBS) 10-item checklist was used to detect presence and degree of unilateral neglect in everyday situations as it is described in [1,5]. Total rating of neglect severity is as following: 0 -No neglect, 1-10 - mild neglect, 11-20 moderate and 21-30- severe neglect.

\section{Results and Discussion}

Table 1. Results in $L B, B T, C C, C M, R O C$ and ROM.

\begin{tabular}{|c|c|c|c|c|}
\hline \multirow[b]{2}{*}{ Task } & \multirow[b]{2}{*}{ Errors in task performance } & \multicolumn{3}{|c|}{ Scores in 3 sessions } \\
\hline & & $\begin{array}{l}\text { First } \\
\text { session }\end{array}$ & $\begin{array}{l}\text { Second } \\
\text { session }\end{array}$ & $\begin{array}{l}\text { Third } \\
\text { session }\end{array}$ \\
\hline \multirow{2}{*}{ LB } & $\begin{array}{l}\text { Displacement of the bisection mark } \\
\text { toward the left }\end{array}$ & 0 & 1 & 1 \\
\hline & $\begin{array}{l}\text { Displacement of the bisection mark } \\
\text { toward the right }\end{array}$ & 2 & 2 & 2 \\
\hline \multirow{2}{*}{ BT } & Elements omitted on the left side & 3 & 4 & 1 \\
\hline & Elements omitted on the right side & 0 & 0 & 0 \\
\hline \multirow{2}{*}{$\mathrm{CC}$} & Elements omitted on the left side & 0 & 0 & 0 \\
\hline & Elements omitted on the right side & 0 & 0 & 0 \\
\hline \multirow{2}{*}{$\mathrm{CM}$} & Elements omitted on the left side & 4 & 0 & 0 \\
\hline & Elements omitted on the right side & 0 & 0 & 0 \\
\hline \multirow{2}{*}{ ROC } & Elements omitted on the left side & 1 & & \\
\hline & Elements omitted on the right side & 0 & & \\
\hline \multirow{2}{*}{ ROM } & Elements omitted on the left side & 7 & & \\
\hline & Elements omitted on the right side & 2 & & \\
\hline
\end{tabular}

Out of the total of 30 attempts in 3 sessions, 2 
displacements of no more than $5 \mathrm{~mm}$ of the bisection mark from the center toward the left and 6 displacements of 7-8 $\mathrm{mm}$ from the center toward the right were registered in LB. Errors in the each session of LB performance, in particular, the number of displacements of the bisection mark from the center either to the right ( 2 errors) or to the left (1 error) does not exceed the normative frames for healthy subjects [27]. Therefore, results in LB do not confirm HN diagnosis.

All the omissions in the BT were registered in the left part of the sample. Number of omissions in the three BT sessions make up to $2.8,11.4$ and $2.8 \%$ of the total of 35 targets to be cancelled respectively. According to accepted standard [14] results of BT do not confirm the diagnosis of $\mathrm{HN}$.

No mistakes (omission of details, distortion of the figure) were registered in CC.

Results in CM are shown in the Figure 1. In the first session patient omitted 4 details on the left side. In the two consecutive sessions patient was able to represent all elements of the sample.

One element was omitted on the left side of figure in ROC, while the number of omissions on the left side of figure increased markedly in ROM (Figure 2). Drawing stile was evaluated as a second level performance: patient started with

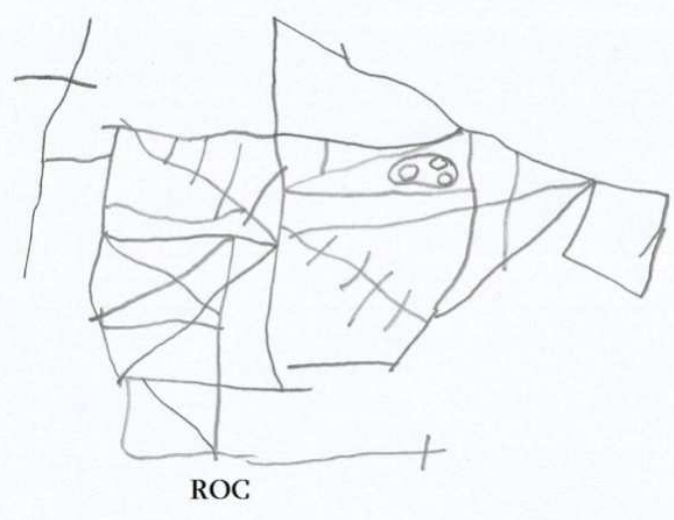

copying triangles, then turned to small details and afterwards tried to connect parts and represent an overall shape of the figure.

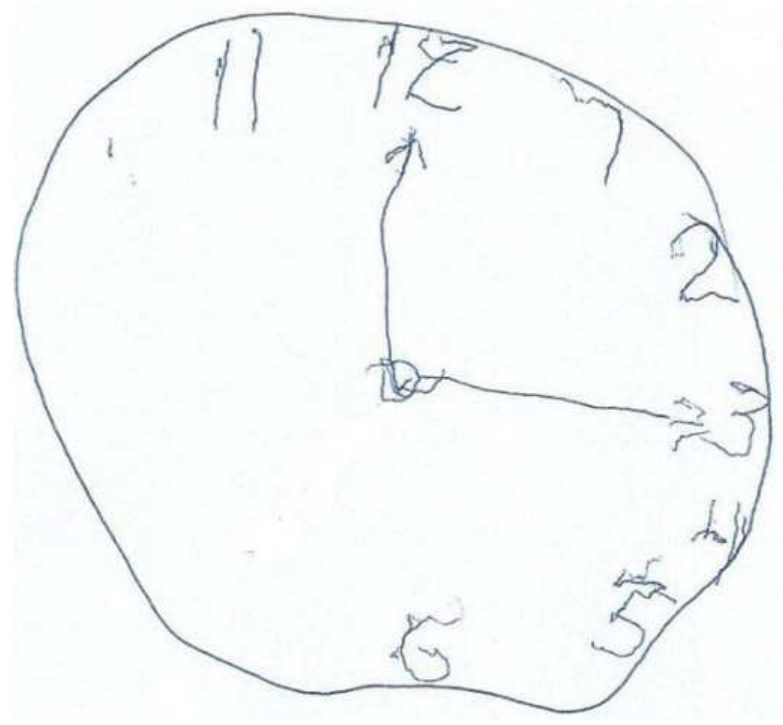

Figure 1. Clock drawing from memory.

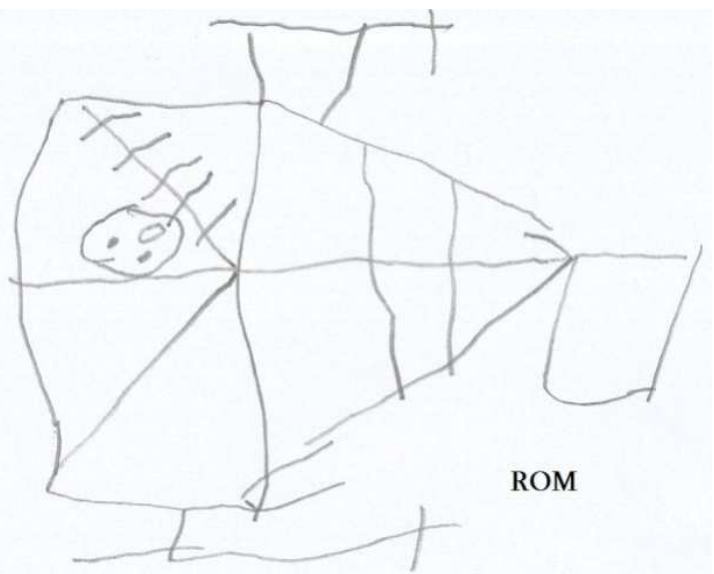

Figure 2. Drawing of Rey-Osterrieth Figure from the sample (ROC, Left) and from memory (ROM, Right).

Only one item in CBS ("Has difficulty in paying attention to noise or people addressing him from the left") was scored 2 in all the three sessions of observation.

Second level of Rey-Osterrieth figure drawing is typical to patients with left hemisphere lesion accompanied with aphasia. Left hemispheric aphasic patients use an analytical strategy, copying the model piecemeal, while patients with righthemispheric lesion as well as lefthemispheric nonaphasic patients use global strategy of representing general configuration and afterwards details of the figure [4]. At the same time, only one omission in ROC is not enough to confirm the HN diagnosis.

Marked increase in the number of omited details in CM and ROM suggests the incidence of uilateral neglect in representational sphere. It is well established, that neglect can be observed, not only in the perceptual domain, but also at a representational level [7]. Therefore, leftsided omissions in the $\mathrm{CM}$ and ROM may be ascribed to the $\mathrm{HN}$ at a representational level. Data obtained coincide with the report on the left neglect in representational domain in patient with the damage to the left hemisphere [11]. It is worth mentioning, however, that patient in [11] suffered from ischemic stroke to the posterior part of the left brain hemisphere, while in the current study, patient was diagnosed as having stroke to the frontal and parietal lobes of the left hemisphere. Fast recovery in $\mathrm{CM}$ in the second and third sessions confirms that neglect after the damage to the left hemisphere recovers rapidly [13].

As for CBS, score 2 suggests mild neglect, limited to inattention to people, addressing from the left.

Recently authors [9] reported on the $\mathrm{HN}$ in result of the repetitive transcranial magnetic stimulation (rTMS) of the intraparietal sulcus. Rightward shift of attention was registered in case of the rTMS of the right intraparietal sulcus. Leftward shift of attention was registered in case of the rTMS to the left intraparietal sulcus. At the same time, leftward shift of attention in case of the leftsided rTMS was registered in $60 \%$ 
of subjects, while $40 \%$ displayed rightward shift. These data may explain the sparcity of clinical cases of leftsided $\mathrm{HN}$ as compared to the incidence of rightsided $\mathrm{HN}$ after the damage to the left hemisphere.

It is well established, that brain hemispheres interact via commissural callosal pathway. Authors [9] advocate the dual account of callosal function in spatial attention, suggesting attention to be organized by both inhibitory as well as facilitatory interaction of the brain hemispheres via the corpus callosum. Structural individual variability within the callosal system is suggested to determine which process, inhibition or facilitation dominates in each particular case. Along with variety of cortical and subcortical structures participating in organization of attention, structural variability of callosal system may play a role in heterogenity of neglect syndrom in each individual clinical case.

In our opinion, results obtained in the current study fit dual account hypothesis. Right hemisphere dominates in organizing spatial attention. However, left hemisphere may contribute to this process by excerting facilitatory influence over the right hemisphere via callosal pathway.

\section{Conclusion}

In the current clinical case, ischemic stroke to the left frontal and parietal lobes is clinicaly expressed in left hemineglect at a representational level. In particular, patient omits details when drawing from memory. At the same time, patient displays difficulty in paying attention to people, addressing him from the left. Hemineglect is suggested due to the interruption of facilitatory influence of the left hemisphere over the right hemisphere in result of the lefthemispheric ischemic lesion.

\section{References}

[1] P. Azouvi, F. Marchal, C. Samuel, L. Morin, C. Renard, A. Louis-Dreyfus, C. Jokie, L. Wiart, P. Pradat-Diehl, G. Deloche, and C. Bergego, "Functional consequences and awareness of unilateral neglect: Study of an evaluation scale", Neuropsychological Rehabilitation, 6, pp. 133-150, 1996.

[2] P. Bartolomeo, M. Thiebaut de Schotten andF. Doricchi, "Left unilateral neglect as a disconnection syndrome," Cerebral Cortex, 17, pp. 2479-2490, 2007.

[3] JM. Beis, C. Keller, N. Morin, P. Bartolomeo, T. Bernati, S. Chokron, M. Leclercq, A. Louis-Dreyfus, F. Marchal, Y. Martin, D. Perennou, P. Pradat-Diehl, C. Prairial, G. Rode, M. Rousseaux, C. Samuel, E. Sieroff, L. Wiart and P. Azouvi, "Right spatial neglect after left hemisphere stroke: qualitative and quantitative study," Neurology, 63, pp. 1600-1605, 2004.

[4] G. Benes, and LP. Pizzamiglio (eds), "Handbook of clinical experimental Neuropsychology," Psychology press, 443, p. $1092,1999$.

[5] C. Bergego P. Azouvi and C. Samuel "Validation d'une echelle d'evaluationfonctionnelle de l'heminegligence dance la vie quotidienne: l'echelle C. B," Annales de Readaptation et de Medecine Physique, 38, pp. 183-189. 1995,
[6] E. Bisiach, "Unilateral Neglect and Related Disorders". In: G. Denes and L. Pizzamiglio, eds. "Handbook of Clinical and Experimental Neuropsychology", Hove, UK: Psychology Press, pp. 479-495, 1999.

[7] E. Bisiach and C. Luzzatti, "Unilateral neglect of representational space," Cortex, 14, pp. 129-133, 1978.

[8] JS. Bloom and GW. Hynd, "The role of the corpus callosum in interhemispheric transfer of information: exitation or inhibition?" Neuropsychol Rev, 15, pp. 59-71, 2005.

[9] M. Chechlacz, GW. Hymphreys, SN. Sotiroupulos, K. Kennard, and D. Cazzoli, "Structural organization of the corpus callosum predicts attentional shift after continous theta burst stimulation," Jornal of Neiroscience, 35, 46, pp. $15353-15368,2015$.

[10] SH. Choi, DL. Na, JC. Adair, SJ. Yoon, CG. Ha and KM. Heilman, "Contralesional directional hypermetria associated with line bisection-specific ipsilesional neglect," Neuropsychologia, 39, pp. 1177-1187, 2001.

[11] G. Cocchini G, A. Bartolo, P. Nichelli, "Left Ipsilesional Neglect for Visual Imagery: A Mental Image Generation Impairment?" Neurocase, 12, pp. 197-206, 2006.

[12] M. Corbetta, MJ Kincade, C Lewis, AZ. Snyder, A. Sapir, "Neural Basis and Recovery of Spatial Attention Deficits in Spatial Neglect", Nat Neurosci, 8, pp. 1603-1610, 2005.

[13] G. Denes, C. Semenza, E. Stoppa, A. Lis, "Unilateral spatial neglect and recovery from hemiplegia: a follow up study". Brain, 105, pp 543-552, 1982.

[14] S. Ferber, HO. Karnath, "How to assess spatial neglect-line bisection or cancellation tasks?" Journal of Clinical and Experimental Neuropsychology, 23, pp. 599-607, 2001.

[15] L. Gauthier, F. Dehaut, Y. Joanette, “The bells test", J ClinInt Neuropsychology, 11, pp. 49-54, 1989.

[16] KM. Heilman, DJ. Adams, "Callosal neglect", Arch Neurol, 60 (2), pp. 276-279, 2003

[17] KM. Heilman, D. Bowers, E. Valestein, RT. Watson. "Hemispace and hemispatialneglect". In: M. Jeannerod, ed. "Neurophysiological and neuropsychological aspects of spatial neglect," Amsterdam: Elsevier, pp. 115-150, 1987.

[18] KM. Heilman, RT. E. Watson, E. Valenstein, "Neglect: Clinical and anatomicaspects." In: Feinberg TE, Farah MJ, eds. "Behavioral Neurology and Neuropsychology", New York: Mc Graw-Hill, pp. 309-318, 1997.

[19] L. Johannsen, HO. Karnath, "How efficient is a simple copying task to diagnose spatial neglect in its chronic phase?" J. Clin. Exp. Neuropsychol, 26, pp. 251-256, 2004.

[20] H-O. Karnath, J Zihl, “Disorders of spatial orientation”. In: T. Brandt, LR. Caplan, J. Dichgans, HC. Diener, C. Kennard eds. "Neurological disorders: course and treatment", San Diego, CA: Academic Press, pp. 277-286, 2003.

[21] RB. Katz, S. Sevush, "Positional dyslexia. Brain and language", 37, pp. 266-289, 1989.

[22] M. Kim, SL. Na, GM. Kim, JC. Adair, KH. Lee, KM. Heilman, "Ipsilesional neglect: behavioural and anatomical features". Journal of Neurology, Neurosurgery and Psychiatry, 67, pp. 35-38, 1999. 
[23] M. Kinsbourne, "Hemi-neglect and hemisphere rivalry" In: EA. Weinstein, RP. Friedland eds. "Hemi-inattention and hemisphere specialization", New York: Raven, pp. 41-49, 1977.

[24] M. Kinsbourne, "Neurophysiological and neuropsychological aspects of spatial neglect". In: M. Jeannerod ed. "Mechanisms of Unilateral neglect", Amsterdam: Elsevier Science, pp. 6986, 1987.

[25] JT. Kleinman, M. Newhart, C. Davis, J. Heidler-Gary, RF. Gottesman, AE. Hillis, "Right hemispatial neglect: Frequency and characterization following acute left hemisphere stroke," Brain Cogn, 64 (1), pp. 50-59, 2007.

[26] SE. Kwon, \& KM. Heilman, "Ipsilateral neglect in a patient following a unilateral frontal lesion", Neurology, 41, pp. 2001-2004. 1991.

[27] M. Mennemeier, E. Vezey, A. Chatterje, SZ. Rapcsak, KM. Heilman, "Contribution of the left and right hemispheres to line bisection”, Neuropsychologia, 35, pp. 703-715, 1997.

[28] A. Parton, P. Malhotra, M. Husain, "Hemispatial neglect", J Neurol Neurosurg Psychiatry, 75, pp. 13-21, 2004.

[29] O. Spreen, E. Strauss (eds), "A compendium of neuropsychological tests", 2-nd edition, Oxford Univ Press, pp. 736-342, 1998.

[30] D. Taylor, "Measuring mild visual neglect: Do complex visual tests activate rightward attentional bias?" New Zealand: Journal of Physiotherapy, 31 (2), pp. 67-72, 2003. 